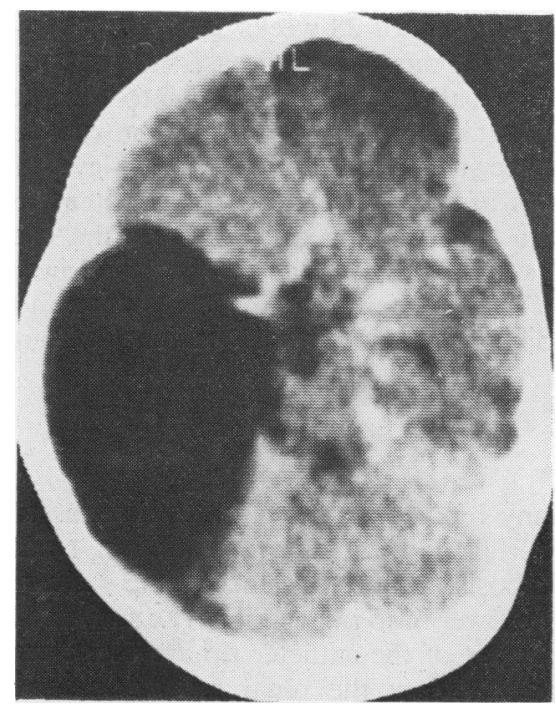

Figs 1 and 2. CT scan of the patient's head showing extensive cerebral defects on the right hemisphere.

Chest $x$-ray, intravenous pyelogram, and urine vanylmandelic acid were normal. Electroencephalogram showed asymmetry with slow-waves over the right occipito-temporal area. A computerised tomography (CT) scan showed extensive defects of the right occipital and temporal lobes compatible with porencephalic cysts (Figs 1 and 2).

\section{Discussion}

No central nervous system malformations associated with amniocentesis have been reported in live newborn babies. Subdural haemorrhage and damage of brain tissue caused by amniocentesis has been found at necropsy in a stillborn baby. ${ }^{2}$ The presence at birth of the 2 subcutaneous nodules overlying the cerebral defects suggests that the amniocentesis needle might have been responsible for the brain lesions. The mechanism of porencephalic cyst formation is not clear. Intracerebral haemorrhage however, could have led to cyst formation.

Although second trimester amniocentesis appears to be a fairly safe procedure, it can lead to serious fetal trauma, ${ }^{3}$ although it is rare. It is obvious that the rare complications do not outweigh the value of amniocentesis in prenatal diagnosis of $\beta$-thalassaemia. ${ }^{4}$ This is particularly true in countries such as Greece where $\beta$-thalassaemia trait is common.

\section{References}

1 National Institute of Child Health and Human Development (NICHD): National Registry for Amniocentesis Study Group. Mid-trimester amniocentesis for prenatal diagnosis. Safety and accuracy. JAMA 1976; 236: 1471-6.

2 Creasman W T, Lawrence $\mathbf{R}$ A, Thiede $\mathbf{H ~ A . ~ F e t a l ~}$ complications of amniocentesis. JAMA 1968; 204: 949-52.

3 Robinson A, Bowes W. Droegemueller W, et al. Intrauterine diagnosis. Potential complications. Am J Obstet Gynecol 1973; 116: 937-41.

4 Leonard C, Kazazian H, Jr. Prenatal diagnosis of hemoglobinopathies. Pediatr Clin North Am 1978; 25: 631-42.

Correspondence to Dr S Youroukos, First Department of Paediatrics of Athens University, Aghia Sophia Children's Hospital, Athens 608, Greece.

\title{
Gluteal skin necrosis after umbilical artery catheterisation
}

\author{
NICHOLAS P MANN \\ Department of Neonatal Medicine and Surgery, The City Hospital, Nottingham
}

SUMMARY Three infants are reported who developed gluteal skin necrosis as a result of umbilical arterial catheterisation. It is important to examine the buttocks and back of every baby catheterised, so that early remedial action can be taken.

Indwelling umbilical arterial catheters are used extensively in neonatal intensive care. Originally they were inserted for the purpose of arterial blood sampling and the infusion of fluids, but their use has been extended to include the continuous measurement of arterial $\mathbf{P o}_{2}$ and blood pressure. It is well known that umbilical arterial catheterisation is associated with ischaemic complications as a result of thrombosis, embolism, or arterial spasm. ${ }^{1}$ I report 3 cases of an unusual complication-gluteal skin necrosis.

\section{Case reports}

Case 1. A boy of 27 weeks' gestation weighing $820 \mathrm{~g}$ 
developed recurrent apnoeic spells which were treated initially with oxygen, and then with intubation and mechanical ventilation. At age 8 hours a 3.5 FG Argyle catheter was inserted into the right umbilical artery without difficulty. Arterial blood was easily obtained and an $x$-ray showed the tip to be in the aorta at the level of L3. Six hours later extensive discoloration of the whole of both buttocks and lower back up to the level of L2 was observed and the catheter was removed immediately. However, the skin became gangrenous; the baby was managed conservatively without skin grafting. Eight months later he has loss of subcutaneous fat and some skin scarring over both buttocks, but no evidence of sciatic nerve involvement.

Case 2. A $1 \cdot 10-\mathrm{kg}$ boy of 32 weeks' gestation developed recurrent apnoeic spells. He was given oxygen initially but later required mechanical ventilation. A 3.5 FG catheter was inserted into the left umbilical artery 2 hours after birth when the baby was well. An $x$-ray showed the tip of the catheter to be at L3-L4 level. The buttocks were inspected $30 \mathrm{~min}$ after insertion and a small area of ischaemic skin was present over the left buttock and left upper outer thigh. The catheter was removed immediately and there was complete healing in a few days without scarring.

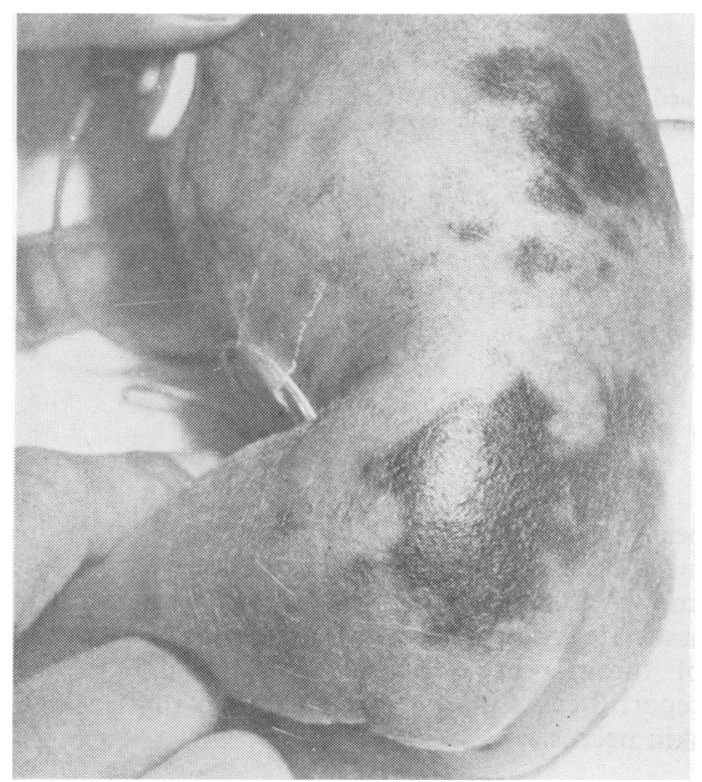

Fig. 1 (Case 3). Evidence of left gluteal and lower back skin necrosis. Complete healing took place within 10 days.
Case 3. A 1 10-kg girl of 28 weeks' gestation developed severe hyaline membrane disease shortly after birth. A 3.5 FG catheter was inserted into the left umbilical artery at age $2 \frac{1}{2}$ hours. An $x$-ray showed the catheter tip to be situated in the aorta at the level of L2. 45 minutes after insertion there was extensive superficial skin necrosis covering most of the left buttock and small patchy areas on the left lower back (Fig. 1). The catheter was removed at once and within a few days there was complete healing without scarring.

\section{Discussion}

There are many reports of the vascular hazards of umbilical arterial catheterisation-such as renal artery thrombosis, superior mesenteric artery thrombosis, or lower limb ischaemia. ${ }^{2}$ The suggested sites for placement of the catheter tip are the lower abdominal aorta below the renal and mesenteric vessels, or just above the diaphragm in the thoracic aorta. In these three infants of very low birthweights, the aim was to place the catheter tip in the lower aorta. The position was checked by $x$-ray after insertion. In Cases 1 and 2 the catheter tip was in a good position at L3 level, in Case 3 it was higher at the level of L2. All three catheters were removed because of gluteal skin necrosis.

The blood supply to the skin of the upper thigh, buttock, and sciatic nerve is derived from the inferior gluteal artery. The superior gluteal artery supplies part of the lower back. These arteries may have been obstructed by thrombosis, embolism, or vasospasm caused by insertion of a catheter; alternatively the origins of these vessels might have been directly occluded by the catheter itself (Fig. 2). The fact that in Cases 2 and 3 the gluteal skin necrosis was on the same side as the umbilical artery which was catheterised supports this idea. In Case 1 the necrosis was bilateral and much more

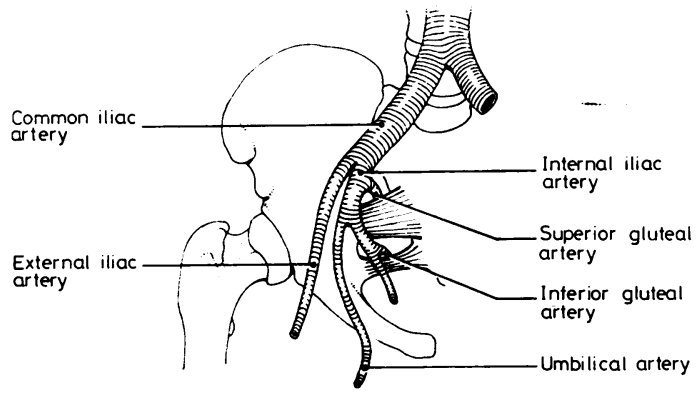

Fig. 2 Relationship between umbilical artery and gluteal arteries. 
extensive. The ischaemia could not have been caused by direct occlusion of the gluteal vessels, but may have resulted from thrombosis in the lower aorta with embolism to both internal iliac vessels. The catheters used were the smallest available but all three babies were of very low birthweights. $10 \%$ dextrose with heparin (500 units in $500 \mathrm{ml}$ ) was slowly infused through the catheter in each case and no drug or other fluid was given.

This complication of umbilical arterial catheterisation has not been widely reported. Cutler and Stretcher reported a baby with skin lesions similar to the lesions of these three infants, 2 days after catheter removal. ${ }^{3}$ Sciatic nerve damage and gluteal necrosis were reported in one baby after placement in the internal iliac artery and injection of multiple drugs, including $25 \%$ dextrose. ${ }^{4}$ Occasionally an aberrant umbilical artery results in placement of the catheter in an end artery-such as the internal pudendal artery-causing perineal skin necrosis. ${ }^{5}$ One baby of a diabetic mother has been reported with gluteal skin necrosis. ${ }^{6}$ It is known that polycythaemia and thrombosis are more common in such infants.

Gluteal skin necrosis in Cases 2 and 3 was diagnosed early because the buttocks and back were inspected routinely shortly after umbilical arterial catheterisation. The catheters were removed at once and there was complete healing. Babies who are ill and require ventilatory support are generally nursed supine so that the buttocks and back are not visible. If an umbilical artery catheter has been inserted the nursing staff should inspect the baby's buttocks and back regularly for skin discoloration, in the same way that they look for signs of ischaemia on the baby's legs. If discoloration is discovered, the catheter should be removed at once.

I thank Dr N Rutter for help.

\section{References}

1 Goetzman B W, Stadalnik R C, Bogren H C, Blankenship W J, Ikeda R M, Thayer J. Thrombotic complications of umbilical artery catheters: a clinical and radiographic study. Pediatrics 1975; 56: 374-9.

2 Wigger H J, Bransilver B R, Blanc W A. Thromboses due to catheterisation in infants and children. $J$ Pediatr 1970; 76: 1-11.

3 Cutler V E, Stretcher G S. Cutaneous complications of umbilical artery catheterisation. Arch Dermatol 1977; 113: 61-3.

4 Purohit D M, Abner H C, deVito P C. Gluteal necrosis with foot drop. Am J Dis Child 1978; 132: 897-9.

5 Rudolph N, Wang H H, Dragutsky D. Gangrene of the buttock. Pediatrics 1974; 53: 106-9.

6 Arad I, Benady W. Gangrene following intra-umbilical in:jection of hypertonic glucose. J Pediatr 1976; 89: 327-8.

Correspondence to Dr N P Mann, Department of Neonatal Medicine and Surgery, City Hospital, Hucknall Road, Nottingham NG5 1PB.

\section{Barrier properties of vernix caseosa}

\section{M JOGLEKAR}

Department of Morbid Anatomy, King's College Hospital, and Department of Neuropathology, Institute of Psychiatry, London

SUMMARY Experiments are described which show that vernix caseosa has a mechanical barrier effect if it is deposited in an unbroken layer. Specific antibacterial properties were not detected in vernix. It is suggested that vernix is best left on a newborn infant because of its mechanical obstruction to bacterial passage.

The nursing care of newborn infants varies from place to place and there has been discussion whether washing off vernix exposes the skin of newborn babies to infection through removal of a substance that may have antibacterial activity. Older studies were in favour of preserving vernix on a newborn baby for its ability to retain heat, especially in premature infants, ${ }^{1}$ and for its anti-inflammatory properties. ${ }^{2}$ Reports on the antibacterial properties of vernix are confined to investigations by Lubinski and Benjamin, ${ }^{3}$ and Sprunt and Redman ${ }^{4}$; neither investigation overcame the difficulty of evenly exposing the bacteria to vernix.

This study was undertaken to determine whether better methods of exposing bacteria to vernix would give different results.

\section{Materials and methods}

Vernix was collected from 9 African babies born in the department of obstetrics at Mulago Hospital, 